\title{
The Flipped Classroom Is Effective for Medical Students to Improve Deep Tendon Reflex Examination Skills: A Mixed-Method Study
}

Shun Uchida ( $\square$ shun.uchid@gmail.com )

Chiba University Hospital

Kiyoshi Shikino

Chiba University Hospital

Kosuke Ishizuka

Chiba University Hospital

Yosuke Yamauchi

Chiba University Hospital

Yasutaka Yanagita

Chiba University Hospital

Daiki Yokokawa

Chiba University Hospital

Tomoko Tsukamoto

Chiba University Hospital

Kazutaka Noda

Chiba University Hospital

Takanori Uehara

Chiba University Hospital

Masatomi lkusaka

Chiba University Hospital

\section{Research Article}

Keywords: clinical skills, confidence, deep tendon reflex, medical student, teaching

Posted Date: January 18th, 2021

DOl: https://doi.org/10.21203/rs.3.rs-139775/v1

License: (c) (i) This work is licensed under a Creative Commons Attribution 4.0 International License.

Read Full License 


\section{Abstract}

Background: Deep tendon reflexes (DTR) are a prerequisite skill in clinical clerkships. However, many medical students are not confident in their technique and need to be effectively trained. We evaluated the effectiveness of a flipped classroom for teaching DTR skills.

Methods: We recruited 83 fifth-year medical students who participated in a clinical clerkship at the Department of General Medicine, Chiba University Hospital, from November 2018 to July 2019. They were allocated to the flipped classroom technique (intervention group, $n=39$ ) or the traditional technique instruction group (control group, $n=44$ ). Before procedural teaching, while the intervention group learned about DTR by e-learning, the control group did so face-to-face. A 5-point Likert scale was used to evaluate self-confidence in DTR examination before and after the procedural teaching ( 1 =no confidence, $5=$ confidence). We evaluated the mastery of techniques after procedural teaching using the Direct Observation of Procedural Skills (DOPS). Unpaired t-test was used to analyze the results of the 5-point Likert scale and DOPS. We assessed self-confidence in DTR examination before and after procedural teaching using a free description questionnaire in the two groups. Additionally, in the intervention group, focus group interviews ( $F G I)$ (7 groups, $n=39$ ) were conducted to assess the effectiveness of the flipped classroom after procedural teaching.

Results: Pre-test self-confidence in the DTR examination was significantly higher in the intervention group than in the control group ( 2.8 vs. 2.3, $P=0.005$ ). Post-test self-confidence in the DTR examination was not significantly different between the two groups (3.9 vs. $4.1, P=0.31)$, and so was mastery (4.3 vs. 4.1, $P=0.68)$. The questionnaires before the procedural teaching revealed themes common to the two groups, including "lack of knowledge" and "lack of self-confidence." Themes about prior learning, including "acquisition of knowledge" and "promoting understanding," were specific in the intervention group. The FGI revealed themes including "application of knowledge," "improvement in DTR technique," and "increased self-confidence."

Conclusions: Teaching DTR skills to medical students in flipped classrooms improves readiness for learning and increases self-confidence in performing the procedure at a point before procedural teaching.

\section{Background}

The deep tendon reflex (DTR) examination is an essential physical examination skill that is assessed in the objective structured clinical examination (OSCE) before clinical clerkships. Even after the OSCE, many medical students are not confident in their skills despite understanding the need and importance of neurological examination. Possible reasons are lack of confidence in the knowledge and performance of the procedure and interpretation of the findings [1]. Inappropriate or inadequate physical examination, including DTR, can result in avoidable adverse events [2]. Thus, effective education about DTR is needed in parallel with a clinical clerkship. 
In this study, the flipped classroom was adopted as a teaching technique. In traditional classes, students are taught in class first. Subsequently, they review the learnings and do the homework. In a flipped classroom, students need to learn before attending the classroom [3]. The flipped classroom emphasizes knowledge utilization through problem-solving and is more effective than traditional classes that accentuate knowledge provision [4]. Based on a revised version of Bloom's taxonomy in cognitive domains [5], we can assign the mastery of procedural knowledge about DTR not only to the level of "remember" but also to a higher level such as "understand" or "apply." We planned to use an e-learning video as material for prior learning. Using video materials has been known to be effective in teaching physical examination [6].

There have been no reports about the usefulness of flipped classrooms in teaching DTR in the literature. We evaluated the usefulness of a flipped classroom compared with a traditional classroom in a mixedmethod study by integrating quantitative and qualitative assessments. We used a 5-point Likert scale to quantitatively evaluate self-confidence in DTR examination before and after the procedural teaching. In addition, we evaluated mastery of techniques after procedural teaching using the Direct Observation of Procedural Skills (DOPS). DOPS is a method of directly observing and assessing techniques performed by learners, and it is frequently used and validated as a method of technique assessment [7]. Learners are expected to acquire higher-order intellectual skills. These skills are difficult to evaluate quantitatively; thus, we assessed learners qualitatively.

\section{Methods}

\section{Trial design}

The flipped classroom was conducted in the intervention group, and the traditional face-to-face classroom was conducted in the control group. The two groups were compared to examine the usefulness of the flipped classroom. The learning objectives of the class were set-participants should be able to derive DTR in five areas: biceps, triceps, flexor brachii, patellar tendon, and Achilles tendon. The participants were allocated to the intervention or control groups. To integrate quantitative and qualitative assessments, we designed a mixed-method study. This study was conducted based on the CONSORT 2010 guidelines [8] (Supplement 1).

\section{Participants}

A total of 83 medical students participated in the study. They were fifth-year students who participated in a clinical clerkship at the Department of General Medicine, Chiba University Hospital, from November 2018 to July 2019. One group consisted of five to six students who practiced for two weeks in this department. All participants learned about basic physical examinations, including DTR in fourth-year classes, and passed a pre-clinical OSCE in which those skills were assessed. 


\section{Intervention}

In the intervention group, participants learned about DTR by seven minutes of e-learning, followed by undergoing ten minutes of procedural teaching. In the control group, participants attended face-to-face classes about DTR and then underwent ten minutes of procedural teaching (Fig. 1). A video available on this website was used as the e-learning material [9]. This video describes DTR, the anatomy of tendons, the locations to blow, and the posture of the examinee. Medical students in the intervention group were able to watch the video repeatedly on their smartphone, tablet, and PC. In the control group, participants underwent face-to-face learning about DTR instead of e-learning. In the interest of fairness, the video was made available for students of the control group after this study.

Three faculty members participated in this study, of which one faculty conducted procedural teaching. The instructional design was conducted considering the opinions of all faculty members, and teaching skills were standardized before the study began.

\section{Outcome measures}

Self-confidence in DTR examination and mastery of the techniques were compared between the two groups. A 5-point Likert scale was used to evaluate self-confidence in the DTR examination before and after the procedural teaching. In the intervention group, the evaluation was performed after prior learning. In the control group, the evaluation was performed before face-to-face teaching. In addition to selfconfidence evaluation, we assessed mastery of the techniques after procedural teaching using the DOPS (Fig. 1). After the procedural teaching, the evaluators directly observed and evaluated the procedures performed on a simulated patient who was played by another student. The item, "technical ability," of DOPS was analyzed. This item evaluates a mastery on a scale of 1 to 6 . The faculty development was conducted to improve inter-observer reliability before this study. In this development, scaling criteria were defined as follows: a score of 3 means the borderline student can derive part of DTR; a score of 4 implies the student can derive all DTR; a score of 2 or less indicates below expectation; and a score of 5 or more means above expectation. To ascertain the baseline, the participants' age, sex, and completion of a clinical clerkship in neurology were investigated. In the intervention group, "accessibility of the prior learning material" was investigated on a 5-point Likert scale for reference to the material's validity.

\section{Sample size}

All fifth-year students who were not yet practicing at the Department of General Medicine participated because this study was also a part of clinical clerkship education in the department. A total of 83 medical students ( 15 groups) participated in the study. This sample size was more than 27 , which is the required sample size for a two-tailed test of the difference in means between the two groups, assuming a significance level of 0.05 , a statistical power of 0.8 , and an effect size of 1.0 . 


\section{Randomization}

The 15 groups were allocated to the intervention or control groups. The allocation was not blinded to the participants or the evaluators. The same person participated as a faculty member and an evaluator.

\section{Statistical method}

All statistical analyses were performed using SPSS Statistics for Windows 26.0 (IBM Co., Armonk, NY, USA). The unpaired t-test, with the significance level set to 0.05 , was used to analyze the results of the 5point Likert scale and DOPS.

\section{Free description questionnaire}

A free description questionnaire was used to qualitatively assess self-confidence in DTR examination before and after procedural teaching. These evaluations were conducted using the same questionnaires with the evaluation of self-confidence. The free description questionnaire was expected to provide explanations for the quantitative results. Opinions about prior learning or flipped classrooms were also collected in the intervention group.

\section{Focus group interview}

In the intervention group, focus group interviews (FGI) $[10]^{-}[11][12]$ were conducted for a qualitative assessment. Considering the study objective, we set the theme to assess the effectiveness of the flipped classroom after procedural teaching. Three evaluators (SU, KS, and KI) took charge as interviewers. The interview guide was prepared after a discussion with the three interviewers (Supplement 2). All students in the intervention group ( $n=39$, seven groups) were interviewed. In the interview, the faculty member interviewed the group that consisted of five to six students after the procedural teaching. The interviewer asked the following question: "Think of the advantage of the flipped classroom. Why do you feel that it was an advantage?" To analyze the interviews, the verbatim reports were created based on the content recorded on a digital voice recorder. These reports were analyzed qualitatively by content analysis. The two researchers (SU, KS) performed the analysis and consensus-building. We performed researcher triangulation to ensure the quality of the analysis. Cohen's kappa coefficient was used to assess interrater reliability [13].

\section{Mixed methods research}

To integrate quantitative and qualitative assessments, a mixed methods study was designed as an exploratory sequential design [14]. The qualitative assessment was intended to support and explain the results of the quantitative assessment as explanations. 


\section{Results}

A total of 39 participants (7 groups) were allocated to the intervention group; 44 participants (8 groups) were allocated to the control group. There was no statistical difference between the two groups in terms of age, sex, and completion of a clinical clerkship in neurology. Each item was analyzed by an unpaired ttest and resulted in the following $P$-values: $P=0.99, P=0.79, P=0.65$. In all participants $(\mathrm{n}=83)$, the mean (SD) age was 23.1 (2.0) years, 80.7\% ( $n=67)$ were male, and the completion of a clinical clerkship accounted for $41.0 \%(n=34)$. In the intervention group $(n=39)$, the mean age was $23.2(2.5)$ years, $79.5 \%$ $(n=31)$ were male, and the completion of a clinical clerkship accounted for $43.6 \%(n=17)$. In the control group $(n=44)$, the mean age was $23.0(1.4)$ years, $81.8 \%(n=36)$ were male, and the completion of a clinical clerkship accounted for $38.6 \%(n=17)$.

Pre-test self-confidence in DTR examination ( $1=$ no confidence, $5=$ confidence) had mean (SD) values of $2.8(0.8)$ and $2.3(0.8)$ in the intervention and control groups, respectively-significantly higher in the intervention group $(P=0.005)$. Post-test self-confidence values in DTR examination were $3.9(0.8)$ and 4.1 $(0.7)$ in the intervention and control groups, respectively, with no significant difference between the two groups $(P=0.31)$. The mastery values evaluated by DOPS were $4.3(0.8)$ and $4.1(0.9)$ in the intervention and control groups, with no significant difference between the two groups $(P=0.68)$ (Table 1$)$.

\section{Table 1. Self-confidence in DTR examinations and DOPS}

\begin{tabular}{|lllc|}
\hline & $\begin{array}{l}\text { Intervention } \\
\text { group } \\
(n=39)\end{array}$ & $\begin{array}{l}\text { Control } \\
\text { group } \\
(n=44)\end{array}$ & $\begin{array}{l}P \\
\text { value }\end{array}$ \\
\hline $\begin{array}{l}\text { Self-confidence in DTR examinations (Pre-test), mean } \\
(S D)^{*}\end{array}$ & $2.8(0.8)$ & $2.3(0.8)$ & 0.005 \\
\hline $\begin{array}{l}\text { Self-confidence in DTR examinations (Post-test), mean } \\
(\text { SD)* }\end{array}$ & $3.9(0.8)$ & $4.1(0.7)$ & 0.31 \\
\hline DOPS, mean (SD) & $4.3(0.8)$ & $4.1(0.9)$ & 0.68 \\
\hline
\end{tabular}

( ${ }^{*}$ : not at all, 2: not confident, 3: confident, 4: very confident, 5: extremely confident)

The free description questionnaires before the procedural teaching revealed themes common to the two groups, including "lack of knowledge" and "lack of self-confidence." Under the theme "lack of selfconfidence," the sub-theme "lack of self-confidence because of poor experience" was extracted (Table 2).

Table 2. Content analysis of pre-practice questionnaire $(n=83)$ 


\begin{tabular}{|lll|}
\hline Free Description & Sub-theme & Theme \\
\hline "I don't know how to perform the procedure well." & Lack of procedural knowledge & $\begin{array}{l}\text { Lack of } \\
\text { knowledge }\end{array}$ \\
\hline $\begin{array}{l}\text { "I have no confidence because I have never } \\
\text { performed the procedure on a patient." }\end{array}$ & $\begin{array}{ll}\text { Lack of self-confidence } \\
\text { because of poor experience }\end{array}$ & $\begin{array}{l}\text { Lack of self- } \\
\text { confidence }\end{array}$ \\
\hline
\end{tabular}

The intervention group comprised the following opinions about prior learning: "I've figured out how to perform the procedure well," "I found the e-learning easy to understand." From these opinions, "acquisition of knowledge" and "promoting understanding" were extracted as themes. In particular, from "promoting understanding," "promotion of understanding using video materials" was extracted as a sub-theme (Table $3)$.

\section{Table 3. Content analysis of pre-practice questionnaire about prior learning in the intervention group $(n=39)$}

\section{Free Description}

"I've figured out how to perform the procedure well."

"I found the e-learning easy to understand."

\section{Sub-theme}

Acquisition of procedural knowledge

Promotion of understanding using video materials
Theme

Acquisition of knowledge

Promotion of understanding

The FGI conducted after the procedural teaching in the intervention group ( 7 groups, $n=39$ ) generated the following opinions: "I'm glad I had the opportunity to practice the techniques I learned," "It was great to get instruction while practicing the technique," and "I think the technique has become more reliable." From these opinions, "application of knowledge," "improvement in DTR technique," and "increased selfconfidence" were extracted as themes (Table 4).

\section{Table 4. Content analysis of post-practice FG* in the intervention group (7 groups, $n=39$ )}

\section{Reaction}

"I'm glad I had the opportunity to practice the techniques I learned."

"It was great to get instruction while practicing the technique."

"I think the technique has become more reliable."

\section{Sub-theme}

Application of procedural knowledge

Improving DTR techniques through instruction

Increased self-confidence in DTR technique

\section{Theme}

Application of knowledge

Improving DTR technique

Increased selfconfidence

The inter-rater reliability was high (Cohen's kappa coefficient $=0.84)$. In the intervention group, "accessibility of the prior learning material" was investigated, and the mean score (SD) was $3.7(0.2)$ ( $1=$ hard to use, $5=$ easy to use). 


\section{Discussion}

Teaching DTR skills to medical students in flipped classrooms improves their readiness for learning and increases their self-confidence in performing the procedure at a point before procedural teaching. If the time for procedural teaching is secured, the mastery of DTR will not decline without face-to-face teaching of DTR knowledge, which may lead to further securing of time for procedural teaching.

In the intervention group, pre-test self-confidence in the DTR examination was significantly higher than that in the control group. We extracted the theme "promoting understanding" from the free description questionnaires before the procedural teaching in the intervention group. This theme suggested that the increased self-confidence was a result of sufficient readiness for learning by "understand," which is a precondition of "apply" in Bloom's taxonomy. Readiness for learning is one of the preconditions of selfdirected learning and has a significant impact on learning motivation [15]. Readiness for learning tends to be enhanced in the flipped classroom because a learner can use prior learning materials at any time or place. Consequently, the time for active learning in the classroom is secured, and the learner's understanding is deepened [16].

If the time for procedural teaching is secured, the mastery of DTR will not decline without face-to-face teaching about DTR knowledge, which may lead to further securing of time for procedural teaching. There were no significant differences between the two groups in the post-test self-confidence and the mastery of DTR. In the intervention group, prior learning led to securing the time for procedural teaching, which might be why they achieved mastery of DTR equivalent to the control group without face-to-face teaching. This suggests that the flipped classroom could secure more time for procedural teaching by using the time spent on face-to-face teaching.

This study does not reveal the flipped classroom's superiorities in post-test self-confidence or mastery of DTR despite expectations for effective teaching because of two reasons. First, procedural teaching had the same duration between the two groups. Second, the quantitative assessment was evaluated at a lower level of Bloom's taxonomy. In Bloom's taxonomy [5], DOPS evaluated how participants "apply" the procedural knowledge. In a previous study on flipped classrooms, the effectiveness of a flipped classroom was assessed at a higher level of Bloom's taxonomy [17]. The study dealt with face-to-face teaching for medical students and revealed the effectiveness at a higher level such as "analyze." Thus, if we evaluate the performance at the "analyze" or "evaluate" levels, flipped classroom would be significantly effective.

Additionally, the qualitative assessment showed no significant difference in the post-test self-confidence between the two groups. When we analyzed the free description questionnaires before the procedural teaching, the majority reported lack of self-confidence due to poor experience. In Bloom's taxonomy, this means that the experience to "apply" the procedural knowledge is poor. Thus, securing the time for procedural teaching might increase self-confidence. There was no difference in the post-test selfconfidence in the two groups, probably because the duration for procedural teaching was the same. The results of $\mathrm{FGl}$ revealed that securing the time for procedural teaching and applying the knowledge 
contributed to increasing self-confidence and mastery of DTR. Thus, we propose that prior learning could not provide the experience of applying procedural knowledge, but procedural teaching can provide it.

In the investigation of accessibility of the prior learning material, the mean score (SD) was 3.7 (0.2). Thus, prior learning was thought to be conducted without significant problems. From the perspective of learner concentration, the duration of video materials should be less than ten minutes [18]. The adopted elearning material was seven minutes, which was valid. To conduct similar flipped classroom instruction, it is necessary to ensure the quality of prior learning by adjusting the duration of the e-learning material to less than ten minutes to make them more accessible.

\section{Limitation}

There were three limitations to this study. First, the allocation to the two groups could not be blinded to the participants and the faculty members, and subjective bias may have affected the results. Second, the rate of prior learning implementation was not measured. At least, there was a significant difference in pretest self-confidence in the DTR examination, as mentioned above. Third, the participants were medical students at a single institution. Notably, this study's results could be applied to other institutions or residents and interns.

\section{Conclusions}

Teaching DTR skills to medical students in flipped classrooms improves their readiness for learning and increases their self-confidence in performing the procedure at a point before procedural teaching.

\section{Declarations}

\section{Ethics approval and consent to participate}

This research was performed in accordance with the Declaration of Helsinki and approved by the Ethics Review Committee of the Chiba University Graduate School of Medicine (Chiba, Japan). The researchers explained to the participants and obtained their informed and voluntary consent. As the researchers took charge of the faculty members, the conflict of interest could arise between the researchers and the participants (the students). As a countermeasure, the faculty members explained to the students that the study would not be used for university grading.

\section{Clinical trial registration}

This study was registered with the University Hospital Medical Information Network Clinical Trials Registry on 17/07/2020 (Unique trial number: UMIN 000041133). 


\section{Consent for publication}

Not applicable.

\section{Availability of data and materials}

The raw dataset supporting the conclusions of this article is available from the corresponding author upon request.

\section{Competing interests}

The authors have no conflicts of interest directly relevant to the content of this article.

\section{Funding}

Not applicable.

\section{Authors' contributions}

S.U. and K.S. contributed to the study conceptualization and design. S.U., K.S. and K.I. contributed to acquisition of the data, analysis and interpretation of the data. S.U. wrote the main manuscript text and prepared all figure and tables. K.S., K.I., Y.Yamauchi, Y.Yanagita, D.Y., T.T., K.N., T.U., and M.I. reviewd the manuscript.

\section{Acknowledgments}

The authors thank the physicians who participated in the present study.

\section{References}

1. Moore FG, Chalk C. The essential neurologic examination: What should medical students be taught? Neurology. 2009;72(23):2020-3.

2. Verghese A, Charlton B, Kassirer JP, Ramsey M, loannidis JPA. Inadequacies of physical examination as a cause of medical errors and adverse events: A collection of vignettes. Am J Med. 2015;128(12):1322-4.

3. Moffett J. Twelve tips for "flipping" the classroom. Med Teach. 2015;37(4):331-6.

4. Hew KF, Lo CK. Flipped classroom improves student learning in health professions education: $A$ meta-analysis. BMC Med Educ. 2018;18(1):38. 
5. Bloom BS, Englehart MD, Furst EJ, Hill WH, Krathwohl DR. Taxonomy of educational objectives, the classification of educational goals, Handbook l: Cognitive domain. 1st ed. New York, NY: Longmans, Green, and Co; 1956. p. 206.

6. Ramani S. Twelve tips for excellent physical examination teaching. Med Teach. 2008;30(9-10):8516.

7. Norcini JJ, McKinley DW. Assessment methods in medical education. Teach Educ. 2007;23(3):23950 .

8. Schulz KF, Altman DG, Moher D; CONSORT Group. CONSORT 2010 Statement: Updated guidelines for reporting parallel group randomised trials. BMJ. 2010;340:c332.

9. Stanford Medicine 25. Deep Tendon Reflexes [homepage on the Internet]. Stanford University School of Medicine. updated 2019 Sep 3; Cited 25 Jul 2020. https://stanfordmedicine25.stanford.edu/the25/tendon.html. Accessed July 25, 2020. Accessed 25 Jul 2020.

10. Barbour RS. The case for combining qualitative and quantitative approaches in health services research. J Health Serv Res Policy. 1999;4(1):39-43.

11. Malterud K. The art and science of clinical knowledge: Evidence beyond measures and numbers. Lancet. 2001;358(9279):397-400.

12. Côté L, Turgeon J. Appraising qualitative research articles in medicine and medical education. Med Teach. 2005;27(1):71-5.

13. Landis JR, Koch GG. An application of hierarchical kappa-type statistics in the assessment of majority agreement among multiple observers. Biometrics. 1977;33(2):363-74.

14. Creswell JW, Plano Clark VL. Designing and conducting mixed methods research. 3rd ed. Thousand Oaks, CA: SAGE; 2017.

15. Knowles MS. The Adult Learner: A Neglected Species. 2nd ed. Houston: Gulf Publishing Co; 1978.

16. Foon K, Kwan C. Flipped classroom improves student learning in health professions education: $A$ meta-analysis. BMC Med Educ. 2018;18(38):1-12.

17. Morton DA, Colbert-Getz JM. Measuring the impact of the flipped anatomy classroom: The importance of categorizing an assessment by Bloom's taxonomy. Anat Sci Educ. 2017;10(2):170-5.

18. Rana J, Besche H, Cockrill B. Twelve tips for the production of digital chalk-talk videos. Med Teach. 2017;39(6):653-9.

\section{Figures}




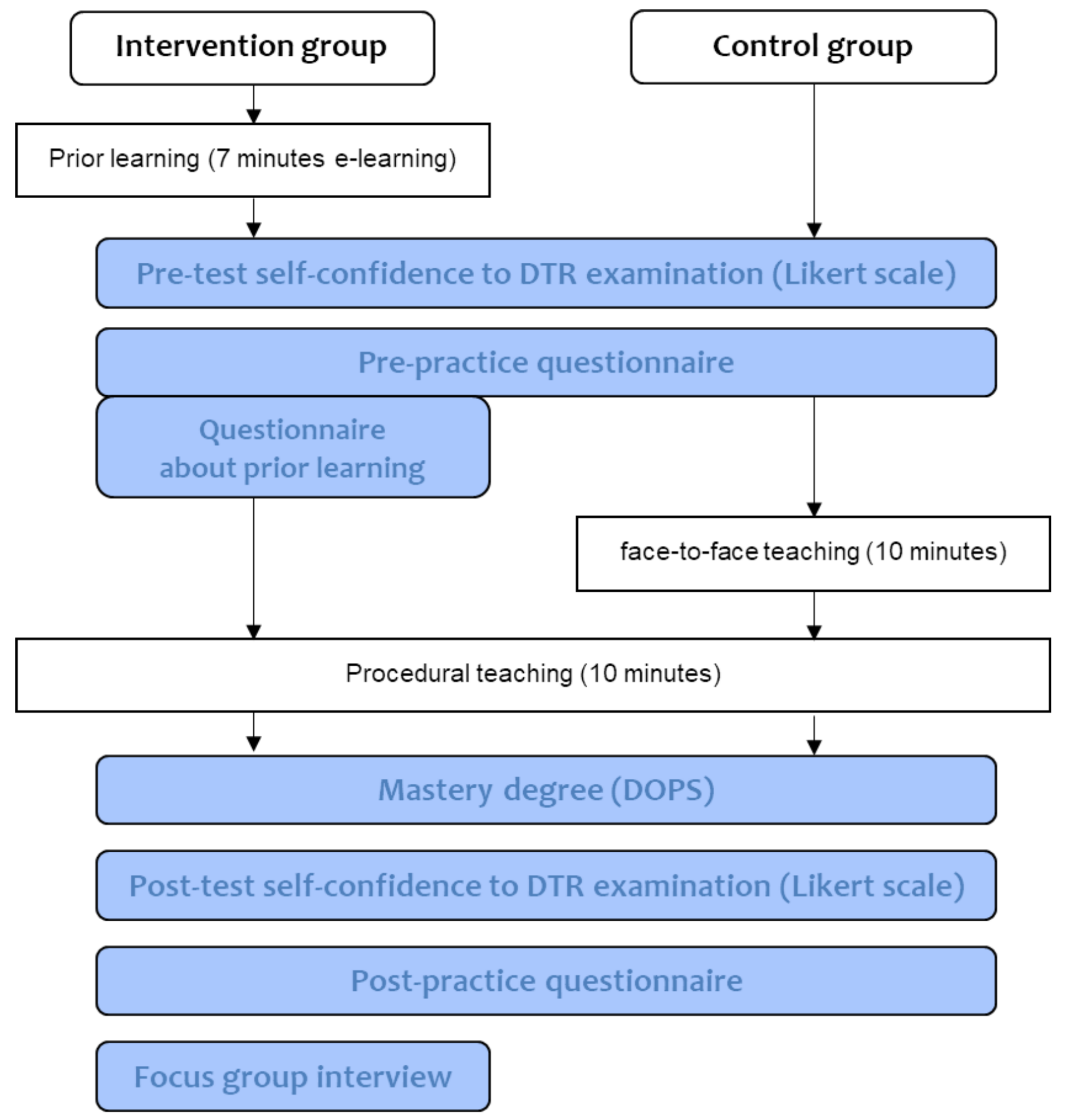

Figure 1

Research flow

\section{Supplementary Files}

This is a list of supplementary files associated with this preprint. Click to download. 
- Supplement1.jpg

- Supplement2.jpg

Page 13/13 